\title{
Modalités de contamination du crustacé planctonique Daphnia magna Straus avec le 134Cs. Etudes de la fixation et de la rétention
}

\section{Contamination modalities of the planktonic crustacean Daphnia magna Straus with ${ }^{134}$ Cs. Uptake and retention studies}

\author{
J. A. Gil Corsico et M. G. Vaz Carreiro
}

Volume 5, numéro 3, 1992

URI : https://id.erudit.org/iderudit/705137ar

DOI : https://doi.org/10.7202/705137ar

\section{Aller au sommaire du numéro}

\section{Éditeur(s)}

Université du Québec - INRS-Eau, Terre et Environnement (INRS-ETE)

ISSN

0992-7158 (imprimé)

1718-8598 (numérique)

\section{Découvrir la revue}

Citer cet article

Corsico, J. A. G. \& Vaz Carreiro, M. G. (1992). Modalités de contamination du crustacé planctonique Daphnia magna Straus avec le 134Cs. Etudes de la fixation et de la rétention. Revue des sciences de l'eau / Journal of Water Science, 5(3), 381-397. https://doi.org/10.7202/705137ar

\section{Résumé de l'article}

Une étude concernant le transfert du césium radioactif dans une chaîne trophique simplifiée d'eau douce, a été réalisée dans le cadre des Contrats

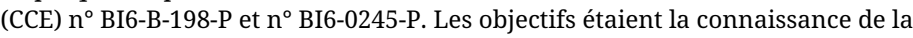
radioécologie du fleuve Tejo (Tage) et le comportement du césium radioactif dans un écosystème naturel. Dans le présent document, sont présentées l'accumulation par voies différentes et la rétention du ${ }^{134} \mathrm{Cs}$ par Daphnia magna Straus (Crustacea, Cladocera).

L'accumulation directe à partir de l'eau du fleuve contaminée avec $\mathrm{du}^{134} \mathrm{Cs}$, a été effectuée avec une concentration initiale, approximative de $10 \mathrm{~Bq} \mathrm{ml}^{-1}$. Le facteur de concentration (FC), rapporté au poids frais des daphnies, a évolué selon la fonction : $F C(t)=57,4\left(1-\mathrm{e}^{-0,275 t}\right)$, avec le temps $(t)$ en jours.

L'élimination du radionucléide est exprimée par la fonction de rétention : $\mathrm{R}(\mathrm{t})$ $=8,5 \mathrm{e}^{-12,211 \mathrm{t}}+91,5 \mathrm{e}^{-0.441 \mathrm{t}}$, qui indique donc deux périodes biologiques, $\mathrm{Tb}_{1}=$ 0,06 jour et $\mathrm{Tb}_{2}=1,6$ jours.

Quand la contamination de D. magna se réalise à partir de la nourriture (Selenastrum capricornutum) contaminée au ${ }^{134} \mathrm{Cs}$,le facteur de transfert trophique suit la fonction : F T $(\mathrm{t})=5,1\left(1-\mathrm{e}^{-0,245 t}\right)$.

Dans ce cas, le processus de rétention est représenté par une fonction exponentielle à un seul terme : $\mathrm{R}(\mathrm{t}) .100 \mathrm{e}^{-0,410 \mathrm{t}}$ et correspond donc à une seule période biologique, $\mathrm{Tb}=1,7$ jour.

La contamination simultanée par les deux vecteurs semble montrer que la voie de contamination la plus importante est le transfert à partir de l'eau. Etant donné que la concentration cellulaire et la concentration en ${ }^{134} \mathrm{Cs}$ de la microalgue S. capricorrrutum étaient approximativement stabilisées, la nourriture n'a été responsable que de $28,5 \pm 8,3 \%$ de la concentration du radionucléide dans D. magna.

En ce qui concerne l'étude de la rétention, on observe que, pour la contamination par l'eau, la période biologique la plus courte doit correspondre à une désorption au niveau des surfaces, tandis que la période la plus longue doit correspondre à l'élimination du ${ }^{134} \mathrm{Cs}$ assimilé. La rétention consécutive à la fixation par l'ingestion de nourriture contaminé, conduit à une seule période biologique, qui ressemble beaucoup à la période longue du premier cas. 


\title{
Modalités de contamination du crustacé planctonique Daphnia magna Straus
}

\author{
avec le ${ }^{134}$ Cs. \\ Etudes de la fixation et de la rétention
}

\author{
Contamination modalities of the planktonic crustacean \\ Daphnia magna Straus with ${ }^{134} \mathrm{Cs}$. \\ Uptake and retention studies
}

\author{
J.A.. GIL CORISCO1, M.C. VAZ CARREIRO1
}

Reçu le 15 mai 1991, accepté pour publication le 20 décembre 1991*.

\section{SUMMARY}

Considering the potential radiological risk, due to the normal operation of the spanish nuclear power plants (Jose CABRERA, TRILLO and ALMARAZ) sited in the Tejo (Tagus) River watershed, the radioecological study of the river is of the utmost interest.

Aiming for a better understanding of radionuclides behaviour in the Fratel dam, a particular freshwater ecosystem in the Tejo River located a few kilometers from the border, several studies have been carried out.

Besides the field follow up, an experimental study conceming the radioactive cesium transfer in a simplified freshwater trophic chain was developed, under the Contracts (CEC) $n^{\circ} B 16-B-198-P$ and $n^{\circ}$ Bl6-0245-P, whose objectives were respectively the knowledge of Tejo River radioecology and the radiocesium behaviour in a natural ecosystem.

In a radiological assessment, the use of site specific data is always recommendable, instead of using default values, what is another perspective of the present work.

The importance of cesium behaviour in the environment, is related to its chemical similarity with potassium, which is a wide-spread element in the living tissues. Foflowing a hypotetical ingestion of radiocesium contaminated foodstuff or water by man, the contamination may then be extended to the whole body, mainly the muscles. Recently, the accident in the ucranian Chernobyl power plant, has alerted the international scientific community to the probiem of the environmental dispersion of this radionuciide.

${ }^{134} \mathrm{Cs}$, a gamma radiation emitter, was the isotope selected for the experiments. Its usual concentration in the radioactive liquid effluents released by PWR plants, is similar to the one of ${ }^{137} \mathrm{Cs}$, an isotope with a hali-life of 30.15

1. Departamento de Protecçāo e Segurança Radiológica, Laboratório Nacional de Engenharia e Tecnologia Industrial, Estrada Nacional 10, 2685 Savavem, Portugal, Tề. (1) 9550021 , Tẻletax (1) 9550117.

* Les commentaires seront reçus jusqu'au 30 mars 1993. 
years. ${ }^{134} \mathrm{Cs}$ has a half-life of only 2.07 years, which is an advantage regarding the production of radioactive wastes resulting from the experiments.

Our objective is to understand the mechanisms of the ${ }^{134} \mathrm{Cs}$ transfer along the trophic chain, and to determine the parameters that might integrate a radioecological model, namely the concentration factors between organisms and water, and the trophic transfer factors between consumers and nourishment.

The simplified trophic chain concerned : a primary producer, the green microalgae Selenastrum capricornutum Printz (CORISCO and CARAEIRO, 1990 a) ; a primary consumer, the planktonic crustacean Daphnia magna Straus ; and a secondary consumer, the omnivorous fish Tinca tinca $L$. (COAISCO and CARREIRO, 1990 b).

In this paper the ${ }^{134} \mathrm{Cs}$ uptake and retention by Daphnia magna Straus, (Crustacea, Cladocera) via different pathways, are presented.

D. magna is a filter-feeding organism very common in freshwater ecosystems and frequently used as a test organism in toxicology. Several authors have been using it, as well, in the field of experimental radioecology. These small crustaceans, hardly exceeding a $3 \mathrm{~mm}$ mesh size, reproduce predominantly by parthenogenesis and, in laboratorial conditions, this process may be permanent if optimum temperature, abundant feeding and water quality are mantained.

The main chemical characteristics of Fratel water, which was used in this study, are presented. The average stable cesium concentration, determined by instrumental neutron activation analysis, is $6 \times 10^{-5} \mathrm{ppm}$.

During the experiments, the temperature was kept at $20 \pm 2{ }^{\circ} \mathrm{C}$ and artificial light was mantained for $15 \mathrm{~h}$ a day, using daylight fluorescent tubes ( 36 watt).

The radiocesium used was a ${ }^{134} \mathrm{Cs}$ chloride in a $0.1 \mathrm{M}$ solution, with a total cesium content of $1 \mu \mathrm{g} \mathrm{ml-1}$.

The radioactive measurements were performed in a well type $11 / 4^{4}$ diameter and $21 / 2^{\prime \prime}$ deep sodium iodine (thatium activated) detector of $4^{\prime \prime} \times 4^{\prime \prime}$, connected to monochannel analyser.

The direct uptake from ${ }^{134} \mathrm{Cs}$ labelled river water was carried out using an initial radionuclide concentration in the water near to $10 \mathrm{~Bq} \mathrm{ml}^{-1}$. The concentration factor (CF), referred to wet weight of daphnids, evolved according to the function : $C F(t)=57.4\left(1-e^{-0,275 t}\right)$, with time $(t)$ referred to days.

When the contamination of $D$. magna was carried out through the nourishment, ${ }^{134}$ Cs labelled Selenastrum capricornutum, the trophic transfer factor (TF) followed the exponential function : $\operatorname{TF}(t)=5.1\left(1-e^{-0,245 t}\right)$.

Combining both pathways simultaneously, water appears to be the main contamination source. The number of cells and 134Cs concentration in microalgae $S$. capricornutum being approximately stabilized, nourishment was responsable for $28.5 \pm 8.3 \%$ of the radionuclide concentration in $D$. magna.

However, using the functions issued from the separated pathways experiments, the assessed contamination of D.magna suggests that the trophic pathway would be predominant.

The evaluation of the relative importance of the contamination pathways, would very likely be dependent on the experimental conditions. Therefore, experimental results must be carefully taken in consideration, and an approach as best as possible to the environmental conditions should be attempted.

The elimination of the radionuclide, after the uptake from water, is expressed by the retention function: $R(t)=8.5 e^{-12.211 t}+91.5 e^{-0.441 t}$, meaning two biolo gical retention compartments with half-lives $T \mathrm{~Tb}_{1}=\mathbf{0 . 0 6}$ day and $\mathrm{Tb}_{2}=\mathbf{1 . 6}$ days. 
Following the uptake from the food pathway, retention is expressed by an exponential with only one term : $R(t)=100 \mathrm{e}^{-0,410 t}$ and, therefore, only one halflife, $\mathrm{Tb}=\mathbf{1 . 7}$ days.

From the retention study after ${ }^{134} \mathrm{C}$ s uptake from water, it appears that the shorter half-life might correspond to the surface desorption, whereas the longer one might correspond to the loss of assimilated ${ }^{134} \mathrm{Cs}$. Following uptake from ingestion of labelled food, retention study lead to a single half-life, very similar to the longer one in the previous case.

These results would suggest that water and food pathways act differently when tested together or separately, and assimilation must be the main concentration mechanism of ${ }^{134} \mathrm{Cs}$ for the crustacean Daphnia magna.

Considering the importance of these planktonic crustaceans as a link in the freshwater trophic chains, the concentration factor at equilibrium $(57 \pm 3)$ and mainly the trophic transfer factor at equilibrium $(5.0 \pm 0.6)$, a significative contamination concerning the ${ }^{134} \mathrm{Cs}$, could be reached.

Key-words : uptake, loss, retention, pathways, crustacea, cladocera, ${ }^{134} \mathrm{Cs}$.

Une étude concernant le transfert du césium radioactif dans une chaîne trophique simplifiée d'eau douce, a été réalisée dans le cadre des Contrats (CCE) $n^{\circ}$ Bl6-B-198-P et $n^{\circ}$ BI6-0245-P. Les objectifs étaient la connaissance de la radioécologie du fleuve Tejo (Tage) et le comportement du césium radioactif dans un écosystème naturel. Dans le présent document, sont présentées l'accumulation par voies différentes et la rétention du ${ }^{134} \mathrm{Cs}$ par Daphnia magna Straus (Crustacea, Cladocera).

L'accumulation directe à partir de l'eau du fleuve contaminée avec du ${ }^{134} \mathrm{Cs}$, a été effectuée avec une concentration initiale, approximative de $10 \mathrm{~Bq} \mathrm{~m} \mathrm{ml}^{-1}$. Le facteur de concentration (FC), rapporté au poids frais des daphnies, a évolué selon la fonction : $F C(t)=57,4\left(1-e^{-0,2751}\right)$, avec le temps $(t)$ en jours.

L'élimination du radionucléide est exprimée par la fonction de rétention : $R(t)=$ $8,5 e^{-12,211 t}+91,5 e^{-0,4411}$, qui indique donc deux périodes biologiques, $\mathrm{Tb}_{1}=$ 0,06 jour et $\mathrm{Tb}_{2}=1,6$ jours.

Quand la contamination de $D$. magna se réalise à partir de la nourriture (Sele nastrum capricornutum) contaminée au ${ }^{134} \mathrm{Cs}$, le facteur de transfert trophique suit la fonction : $\mathbf{T}(t)=5,1\left(1-e^{-0,245 t}\right)$.

Dans ce cas, le processus de rétention est représenté par une fonction exponentielle à un seul terme: $R(t)=100 \varepsilon^{-0,410 t}$ et correspond donc à une seule période biologique, $\mathrm{Tb}=1,7$ jour.

La contamination simultanée par les deux vecteurs semble montrer que la voie de contamination la plus importante est le transfert à partir de l'eau. Etant donné que la concentration cellulaire et la concentration en ${ }^{134} \mathrm{Cs}$ de la microalgue $S$. capricornutum étaient approximativement stabilisées, la noumiture n'a été responsable que de $\mathbf{2 8 , 5} \pm \mathbf{8 , 3} \%$ de la concentration du radionucléide dans $D$. magna.

En ce qui concerne l'étude de la rétention, on observe que, pour la contamination par l'eau, la période biologique la plus courte doit correspondre à une désorption au niveau des surfaces, tandis que la période la plus longue doit correspondre à l'élimination du ${ }^{134} \mathrm{Cs}$ assimilé. La rétention consécutive à la fixation par l'ingestion de nourriture contaminé, conduit à une seule période biologique, qui ressemble beaucoup à la période longue du premier cas.

Mots clés : accumulation, rétention, voies de contamination, crustacea, cladocera, ${ }^{134} \mathrm{Cs}$. 


\section{1 - INTRODUCTION}

Etant donné ie risque radiologique potentiel dû au fonctionnement normal des centrales nucléaires espagnoles situées dans le bassin hydrographique du Fleuve Tejo (Tage), l'étude radioécologique du fleuve présente une grande importance.

Les travaux réalisés ont pour objectif de mieux connaître le comportement du césium radioactif dans l'écosystème d'eau douce de la lagune du barrage de Fratel, dans la partie Portugaise du Tejo (à quelques kilomètres de la frontière).

Outre les études de terrain, une étude expérimentale concernant une chaîne trophique d'eau douce simplifiée (une microalgue, un crustacé planctonique et un poisson omnivore) a été conduite. Le ${ }^{134} \mathrm{Cs}$ a été choisi parce qu'il a joué un rôle très important dans la séquence de l'accident de Chernobyl et de plus, il est présent dans les effluents liquides des centrales nucléaires ayant des réacteurs à eau pressurisée (R.E.P.), à des quantités proches de celles du ${ }^{137} \mathrm{Cs}$.

L'étude du premier niveau trophique, l'accumulation et la rétention du ${ }^{134} \mathrm{Cs}$ par le producteur primaire, la microalgue Selenastrum capricornutum Printz (Chlorophyceae), a déjà été réalisée (CORISCO et CARREIRO, 1990 a).

Cet article présente les résultats relatifs à la contamination par le ${ }^{134} \mathrm{Cs} \mathrm{du}$ deuxième niveau trophique considéré, Daphnia magna Straus (Crustacea, Cladocera), à partir de l'eau, à partir de la nourriture par ingestion de la microalgue $S$. capricomutum, et par ces deux voies simultanées.

L'analyse de la rétention a été réalisée séparément selon que la contamination a été directe ou par l'intermédiaire de la nourriture. Une évaluation de la contribution de la nourriture à la contamination globale du Crustacé est aussi présentée.

Le demier niveau trophique considéré dans l'étude de cette chaîne trophique simplifiée, est le poisson Tinca tinca Linnaeus (CORISco et CARREIRO, 1990 b).

Compte tenu du rôle très important joué par les conditions écologiques sur les transferts des radioéléments, le facteur de concentration et le facteur de transfert trophique sont affectés par beaucoup de paramètres de l'environnement. Aussi pour chaque centrale nucléaire en fonctionnement normal, il est nécessaire de faire une évaluation du transfert vers l'homme, des radioéléments rejetés en utilisant des paramètres spécifiques au site, qui seront plus précis que les valeurs rapportés par la bibliographie. L'expérimentation semble donc se justifier.

Notre objectif est de mieux connaître les transfert du ${ }^{134} \mathrm{Cs}$ dans un chaîne trophique d'eau douce, pouvant aboutir à l'homme, et développer un modèle radioécologique. 


\section{2 - MATÉRIEL ET MÉTHODES}

Daphnia magna Straus (Crustacea, Cladocera) est une des espèces de zooplancton d'eau douce les plus étudiées. Ce crustacé apparaît dans la péninsule ibérique, dans les eaux minéralisées plutôt que dans les eaux eutrophisées (ARMENGOL, 1978 in MARGALEF, 1983). Dans la nature les individus n'atteignent fréquemment que $1-2 \mathrm{~mm}$, mais peuvent atteindre $5 \mathrm{~mm}$, le poids sec variant de 50 à $400 \mu \mathrm{g}$ (MARGALEF, 1983). D. magna se nourrit de petites particules en suspension dans l'eau (jusqu'à $60 \mu \mathrm{m}$ ) qui sont retenues par l'appareil filtrant (BURNS, 1968 in DE PAUW et al., 1981). Dans la nature, la reproduction est caractérisée par une période de parthénogenèse cyclique ; en automne il y a une dernière génération parthénogénétique, composée alors de femelles et de mâles, qui après l'accouplement produisent des œufs de résistance. L'éclosion arrive au printemps suivant et le cycle parthénogénétique recommence (BOUE et CHANTON, 1958 in LAMBRECHTS, 1984).

Les principales caractéristiques chimiques de l'eau de la retenue de Fratel qui a été utilisée dans cette étude, sont présentées dans le tableau 1 (CARREIRO, 1990). La concentration en césium stable déterminée par la technique d'analyse par activation neutronique a été évaluée à $6 \times 10^{-5} \mathrm{ppm}$, (REIS et al., 1988).

L'eau pour les essais a été filtrée sur papier sans cendres, de rétention cristalline (Whatman $n^{\circ} 40$ ).

La température a été maintenue à $20 \pm 2{ }^{\circ} \mathrm{C}$. Une période d'éclairement de $15 \mathrm{~h}$ par jour a été réalisée avec des tubes fluorescents (36 watt) qui reconstituent approximativement le spectre solaire. L'aération et une faible agitation de l'eau dans les flacons, ont été maintenues par injection d'air à l'aide de compresseurs d'aquarium.

Tableau 1 Caractéristiques de l'eau prélevée au barrage de Fratel (1987-1989).

Table 1 Charactenistics of the water from Fratel dam (1987-1989).

\begin{tabular}{|c|c|c|c|}
\hline \multirow[b]{2}{*}{ Eléments } & \multicolumn{3}{|c|}{ Concentration (mg $1^{-1}$ ) } \\
\hline & Valeur moyenne & $\begin{array}{l}V_{b} \\
\text { et } m:\end{array}$ & $\begin{array}{l}\text { les } \\
\text { urées }\end{array}$ \\
\hline $\begin{array}{l}\mathrm{Ca}^{2+} \\
\mathrm{Ma}^{2+} \\
\mathrm{Na}^{+} \\
\mathrm{K}^{+} \\
\mathrm{NH}^{4+} \\
\mathrm{Cl}^{4} \\
\mathrm{SO}_{4}^{2-} \\
\mathrm{NO}_{3}^{2-} \\
\mathrm{NO}_{2}^{-} \\
\mathrm{P}_{2} \mathrm{O}_{4}^{-} \\
\mathrm{Fe} \text { total } \\
\mathrm{P} \text { total } \\
\mathrm{N} \text { total }\end{array}$ & $\begin{array}{c}44,00 \\
12,0 \\
20,3 \\
2,69 \\
0,299 \\
28,8 \\
58,8 \\
1,05 \\
0,003 \\
0,35 \\
0,116 \\
0,564 \\
3,31\end{array}$ & $\begin{array}{c}25,0 \\
7,9 \\
9,3 \\
0,90 \\
0,034 \\
11,0 \\
25,0 \\
0,272 \\
0,002 \\
0,88 \\
0,029 \\
0,38 \\
0,92\end{array}$ & $\begin{array}{c}67,0 \\
22,0 \\
27,0 \\
6,5 \\
0,772 \\
48,0 \\
97,0 \\
2,46 \\
0,014 \\
0,32 \\
0,34 \\
0,95 \\
12,0\end{array}$ \\
\hline $\begin{array}{c}\text { Dureté }\left(\mathrm{mg} \mathrm{1}^{-1}\right) \\
\mathrm{pH}\end{array}$ & $\begin{array}{r}133,0 \\
7,8\end{array}$ & $\begin{array}{r}56,0 \\
6,9\end{array}$ & $\begin{array}{r}238,0 \\
8,8\end{array}$ \\
\hline
\end{tabular}


Le césium radioactif utilisé est le ${ }^{134} \mathrm{Cs}$ sous forme de chlorure en solution $0,1 \mathrm{M}$ de $\mathrm{HCl}$, dont la concentration en césium total est de $1 \mu \mathrm{g} \mathrm{m}^{-1}$.

\section{Contamination par l'eau}

La contamination directe à partir de l'eau a été réalisée en utilisant une radioactivité initiale d'approximativement $10 \mathrm{~Bq} \mathrm{ml}^{-1}$ et 200 femelles, de taille supérieure à $1,5 \mathrm{~mm}$, dans 31 d'eau. A chaque temps d'échantillonnage on a pris tous les individus présents, nouveau-nés compris, en les répartissant en deux échantillons similaires. La période de contamination a été de 18 jours et, pendant ce temps, les crustracés n'ont pas reçu de nourriture. Deux essais de cette expérience ont été réalisés simultanément.

Après la période de contamination, les daphnies des deux flacons ont été transférées dans $3 \mathbf{I}$ d'eau de Fratel non contaminée et nourries avec des microalgues également non contaminées, pour réaliser l'expérience de désorption et l'analyse de la rétention. Cinq fois par semaine le milieu de culture a été complètement renouvelé, pour empêcher la reconcentration dans les crustacés, du radionucléide excrété et remis en solution. A chaque temps d'échantillonnage, on a mesuré la radioactivité sur la totalité des individus répartis en deux échantillons.

\section{Contamination par la voie trophique}

Pour l'étude de la contamination indirecte, c'est-à-dire par la voie trophique, on a nourri les daphnies, initialement 200 dans 31 d'eau non radioactive, avec des microalgues contaminées par le ${ }^{134} \mathrm{Cs}$. Cinq fois par semaine on a transféré tous les crustacés présents, les nouveau-nés aussi, dans un nouveau flacon avec de l'eau non radioactive et de la nourriture (algues) contaminée, dont la concentration en ${ }^{134} \mathrm{Cs}$ a été déterminée.

Les microalgues fournies comme nourriture, ont été séparées de leur milieu par centrifugation, puis elles ont été rincées dans de l'eau distillée et centrifugées, deux fois consécutivement. Un échantillon de ce dépôt algal a été déposé sur une membrane filtrante $(0,45 \mu \mathrm{m})$ pour mesurer la radioactivité (CORISCO et CARREIRO, 1990 a). Le reste du dépôt algal a été remis en suspension dans l'eau.

Le rinçage des microalgues a pour but d'entraîner la désorption du ${ }^{134} \mathrm{Cs}$ adsorbé sur les membranes cellulaires, ne laissant que la fraction assimilée, laquelle représente $20 \%$ du total du radionucléide fixé (CORISCO et CARREIRO, $1990 \mathrm{a})$. Au fur et à mesure que ces microalgues ont été consommées comme nourriture, leur radioactivité a diminué selon la fonction $a_{0} e^{-1 \cdot 28 t}$ ( $t$ en jours), $\mathrm{a}_{\mathrm{o}}$ étant la valeur initiale en $\mathrm{Bq} \mathrm{\textrm {g } ^ { - 1 }}$, poids sec. Cette fonction est vérifiée pour des périodes allant jusqu'à deux jours, puisque après cette période dans un milieu confiné, une certaine stabilisation apparaît (CORISCo et CARREIRO, 1990 a). Ce principe a été appliqué aux week-ends, pendant lesquels ni l'eau ni la nourriture des daphnies n'ont été renouvelées.

Afin d'étudier la rétention, on a utilisé les daphnies qui restaient après 25 jours et on a suivi la même procédure que pour la désorption consécutive à la contamination directe. 


\section{Contamination simultanée par l'eau et la voie trophique}

Une troisième modalité de fixation du ${ }^{134} \mathrm{Cs}$ par les voies eau et nourriture simultanées a été suivie. Afin d'obtenir une biomasse initiale d'algues assez grande pour permettre une expérience de 3 à 4 semaines, l'eau de Fratel a été enrichie en sels nutritifs $\left(57,5 \mathrm{mg} \mathrm{l}^{-1}\right.$ de $\mathrm{K}^{+}, 6,75 \mathrm{mg} \mathrm{I}^{-1}$ de $\mathrm{Ca}^{2+}, 3,25 \mathrm{mg}$ $\mathrm{I}^{-1}$ de $\mathrm{Na}^{+}$et $0,41 \mathrm{mg} \mathrm{I}^{-1}$ de $\mathrm{Fe}^{3+}$ ), comme décrit par CoRISCO et CARREIRO (1990 a). Dans deux flacons (cas I et II) contenant chacun 31 d'eau dont la radioactivité était approximativement $20 \mathrm{~Bq} \mathrm{mi}-1$ de ${ }^{134} \mathrm{Cs}$, les daphnies et les microalgues ont été maintenues ensemble. Cependant, les crustacés (200 par flacon) n'ont été introduits que lorsque les microalgues ont atteint l'état d'équilibre (stabilisaton de la croissance), approximativement $4 \times 10^{6}$ cellules $\mathrm{ml}^{-1}$ et $800 \mathrm{~Bq} \mathrm{~g}-1$ (poids sec). Un autre flacon sans les microalgues a été utilisé comme référence, pour déterminer l'influence de l'eau enrichie sur la contamination de $D$. magna et évaluer la contribution de l'ingestion de S. capricornutum dans les cas I et ll.

\section{Echantillonnage et mesures radioactives}

Pour préparer des échantillons, les daphnies ont été recueillies sur filet en nylon de $200 \mu \mathrm{m}$, rincées à l'eau courante, puis séparées des mues sur un tamis, préalablement pesé, adapté aux dimensions du système de détection. Le poids frais a été déterminé, l'eau restante ayant été absorbée d'abord avec du papier filtre (DE PAUW et al., 1981).

La radioactivité de l'eau, liée aux formes solubles (échantillons de $10 \mathrm{ml}$ ), et des particules a été mesurée, l'eau ayant été filtrée, sous vide, sur des membranes Millipore $(0,45 \mu \mathrm{m})$.

Les mesures de radioactivité ont été faites avec un détecteur gamma d'iodure de sodium, à cristal puits, relé à un sélecteur d'amplitude monocanal (les dimensions du cristal étaient $4^{\prime \prime} \times 4^{\prime \prime}$ et celles du puits $1,25^{\prime \prime}$ de diamètre et 2,5 de profondeur).

\section{3 - RÉSULTATS ET DISCUSSION}

\subsection{Contamination par l'eau}

\subsubsection{Fixation}

Au cours de l'expérience, la radioactivité de l'eau dans les deux flacons a montré des valeurs très semblables. On a donc considéré l'ensemble des résultats provenant des deux groupes de daphnies, présentant à chaque temps de mesure, la moyenne statistique de quatre valeurs correspondant à quatre échantillons dont la radioactivité a été mesurée. 
Une semaine après le début de l'expérience, la cinétique de fixation conduit à une stabilisation du facteur de concentration ( $F C)$, qui est le rapport entre la radioactivité des daphnies ( $\mathrm{Bq} \mathrm{g}^{-1}$, poids frais) et la radioactivité de l'eau (Bq $\left.\mathrm{ml}^{-1}\right)$. L'analyse des résultats, effectuée par la méthode proposée par WHICKER et SCHULTZ (1982), montre une cinétique (fig. 1) qui s'exprime par la fonction:

$$
\mathrm{FC}_{(\mathrm{t})}=57,4\left(1-\mathrm{e}^{-0,275 \mathrm{t})}\right.
$$

KING (1964) a déterminé un facteur de concentration de 38 (rapporté au poids frais) pour $D$. magna avec le ${ }^{137} \mathrm{Cs}$, dans un essai de contamination de trois jours, la radioactivité initiale de l'eau étant de $185 \mathrm{~Bq} \mathrm{~m} \mathbf{l}^{-1}$, et la température de $22^{\circ} \mathrm{C}$; à $8^{\circ} \mathrm{C}$ une valeur plus faible, $\mathrm{FC}=29$, a été obtenue. FOULQUIER et al. (1978) utilisant le même radionucléide avec une radioactivité initiale de $1850 \mathrm{~Bq} \mathrm{ml}-1$, et des périodes de contamination plus longues (28 jours), ont évalué un FC de 3,6 (sans référence à la température de l'eau). Finalement LAMBRECHTS (1984) estime un FC de $25 \pm 5$ à l'état d'équilibre, entre le $7^{e}$ et le $17^{\ominus}$ jours, la radioactivité moyenne de l'eau étant de $42 \mathrm{~Bq} \mathrm{ml}-1$.

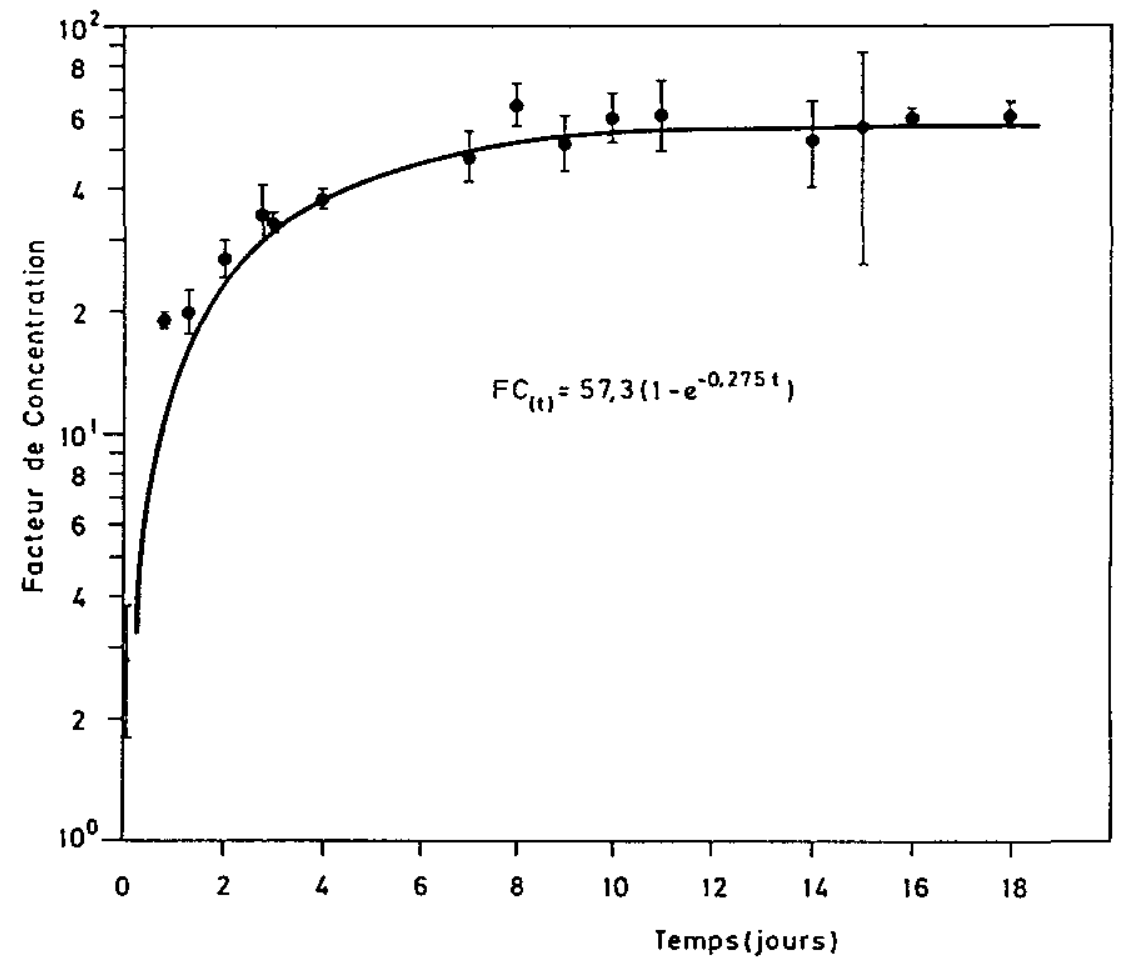

Figure 1 Evolution du facteur de concentration ( $F C$ D. magna ( $\mathrm{Bq} \mathrm{g} \mathrm{g}^{-1}$ poids frais) / eau (Bq $\mathrm{l}^{-1}$ ), pour le ${ }^{134} \mathrm{Cs}$.

Evolution of the concentration factor (FC) D. magna $\left(B q g^{-1}\right.$ fresh weight) / water $\left(\mathrm{Bq} \mathrm{ml}^{-1}\right)$, for ${ }^{134} \mathrm{Cs}$. 


\subsubsection{Rétention}

La concentration initiale du ${ }^{134} \mathrm{Cs}$ dans $D$. magna était de $707 \mathrm{~Bq} \mathrm{~g} \mathrm{~g}^{-1}$ (poids frais). Considérant la variation du pourcentage de rétention, $R_{t}(R=$ concentration initiale/conc. actuelle $\times 100)$, et utilisant la méthode analytique proposée par WHICKER et SCHULTZ (1982), on arrive à une fonction qui s'exprime par la somme de deux exponentielles (fig. 2) :

$$
R_{(t)}=8,5 e^{-12,21}+91,5 e^{-0,441 t}
$$

déterminant deux périodes biologiques, $T b_{1}=0,06$ jour et $T b_{2}=1,6$ jour.

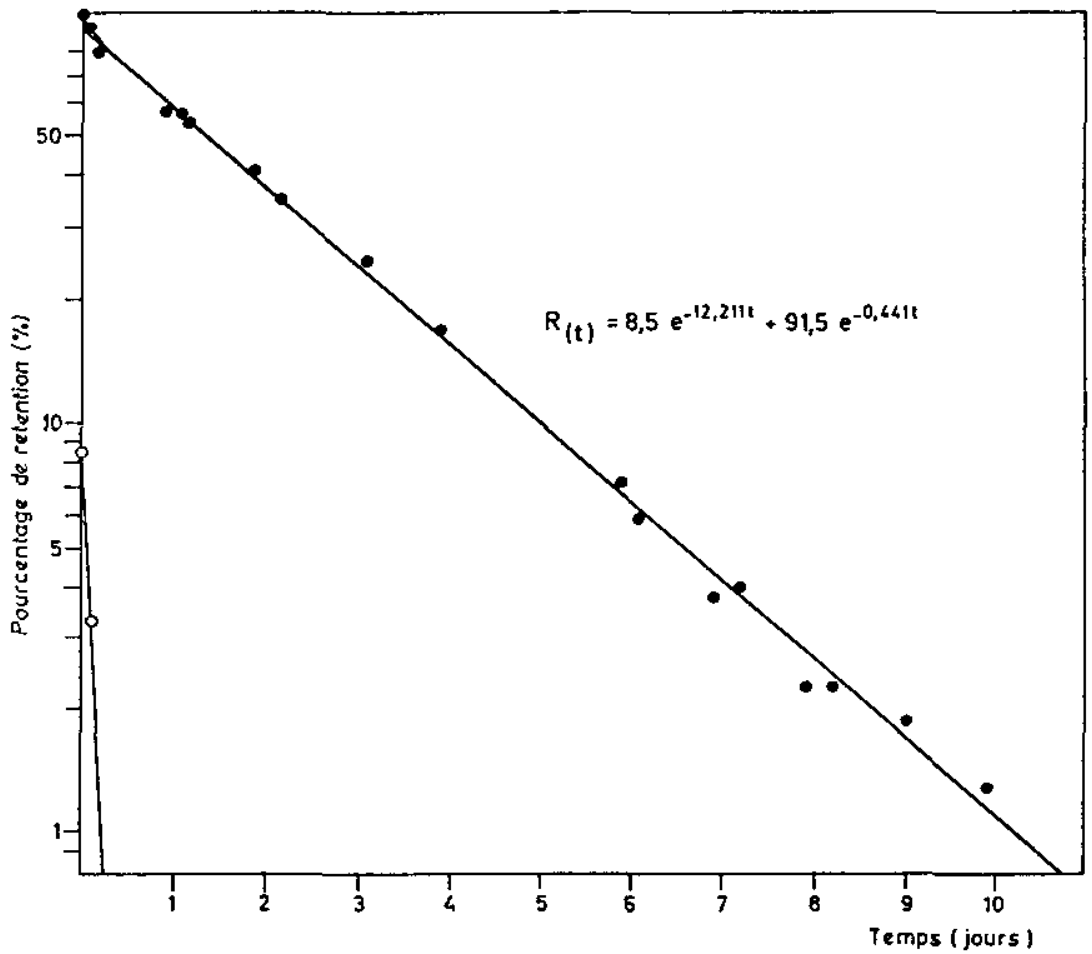

Figure 2 Evolution du pourcentage de rétention du ${ }^{134} \mathrm{Cs}$ par $D$. magna contaminée par l'eau, montrant les deux composantes exponentielles de la fonction.

Evolution of ${ }^{134} \mathrm{Cs}$ retention in D. magna contaminated from water, showing two rate functions.

KING (1964) réfère pour le ${ }^{137} \mathrm{Cs}$ des valeurs de rétention de $73 \%$ et $50 \%$ respectivement, après 24 et 72 heures de désorption, tandis que nous obtenons des valeurs de $59 \%$ et $24 \%$.

Le compartiment à faible rétention ( $8,5 \%$ ), correspondant à la période plus courte, doit être associé aux surfaces exposées au milieu extérieur. Effectivement, les daphnies étant des organismes filtreurs, très probablement l'adsorption sur le système de filtration, dont l'extension est approximativement proportionnelle au carré de la longueur du corps (EGLOFF et PALMER, 1971 in 
MARGALEF, 1983), doit jouer un rôle important. L'autre compartiment montre une rétention plus significative $(91,5 \%)$; cette fraction doit être sous une forme assimilée puisque l'élimination est plus lente.

\subsection{Contamination par la voie trophique}

\subsubsection{Fixation}

Bien que la radioactivité des microalgues disponibles pour les crustacés ait présenté des fluctuations cycliques (fig. 3), une valeur moyenne, de $714 \mathrm{~Bq} \mathrm{~g}-1$ (poids sec), a été prise en compte pendant toute la durée de l'essai.

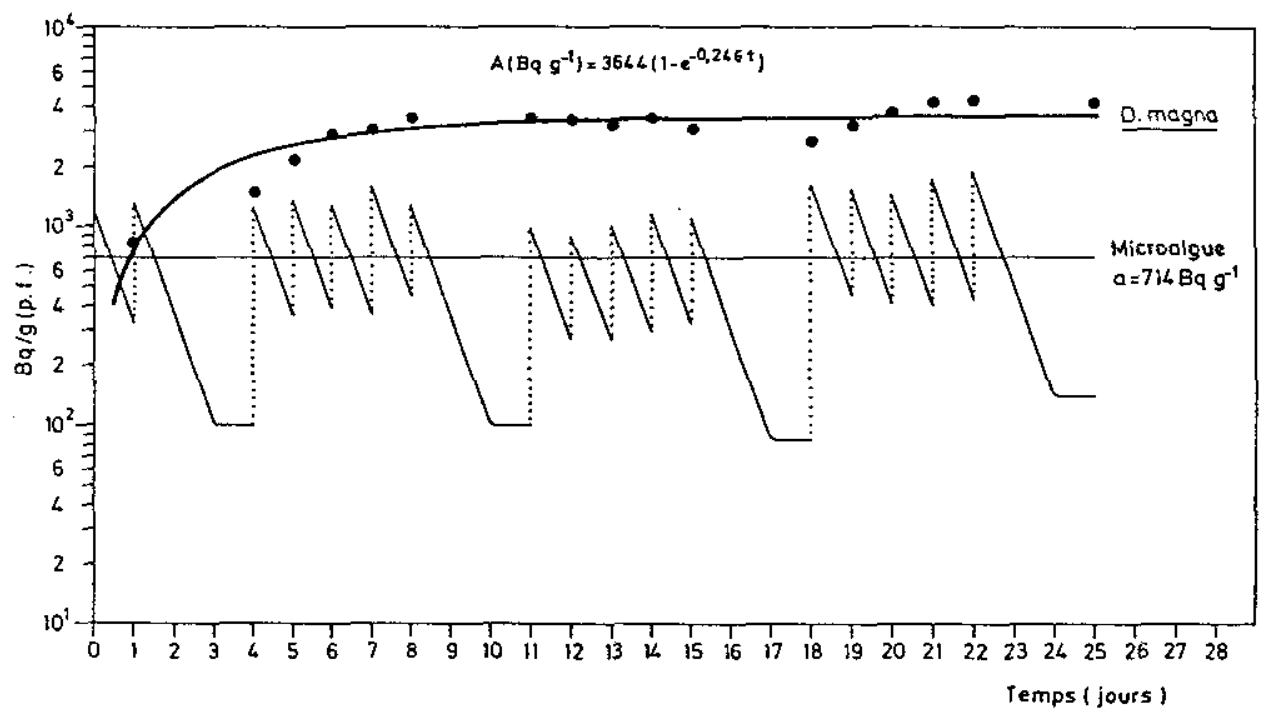

Figure 3 Fixation du ${ }^{134} \mathrm{Cs}$ par (A) D. magna à partir de la nourriture, et fluctuation de la valeur moyenne (a) de la concentration du radionucléide dans la microalgue $S$. capricornutum, utilisée comme nourriture.

${ }^{134} \mathrm{Cs}$ uptake from food by (A) D. magna and fluctuations and mean value (a) of the radionuclide concentration in the microalgae S. capricornutum, used as nourishment.

La radioactivité de $D$. magna a été évaluée d'abord en fonction du poids frais, puis convertie en fonction du poids sec. Le facteur de conversion, 13,89 $\left(O^{\prime}=1,55\right)$ a été antérieurement déterminé, en mesurant la concentration du ${ }^{134} \mathrm{Cs}$ dans 33 échantillons de daphnies avant et après séchage jusqu'à un poids constant ; cette valeur est la moyenne arithmétique des valeurs du rapport radioactivité en poids $s e c /$ radioactivité en poids frais.

La figure 3 montre les fluctuations de la concentration du ${ }^{134} \mathrm{Cs}$ dans la microalgue $S$. capricornutum (nourriture contaminée) et parallèlement l'évolution de la concentration du ${ }^{134} \mathrm{Cs}$ dans $D$. magna, laquelle suit la fonction :

$$
\mathrm{A}=3644\left(1-\mathrm{e}^{-0,246 t}\right) \mathrm{Bq} \mathrm{g}^{-1} \text { (poids sec) }
$$

(t en jours) 
L'évolution du facteur de transfert trophique (FT), évalué par le rapport radioactivité dans $D$. magna $\left(8 q g^{-1}\right.$ p.s.) / radioactivité dans $S$. capricornutum (Bq $\mathrm{g}^{-1}$ p.s.), est présentée sur la figure 4. Le processus suit la fonction :

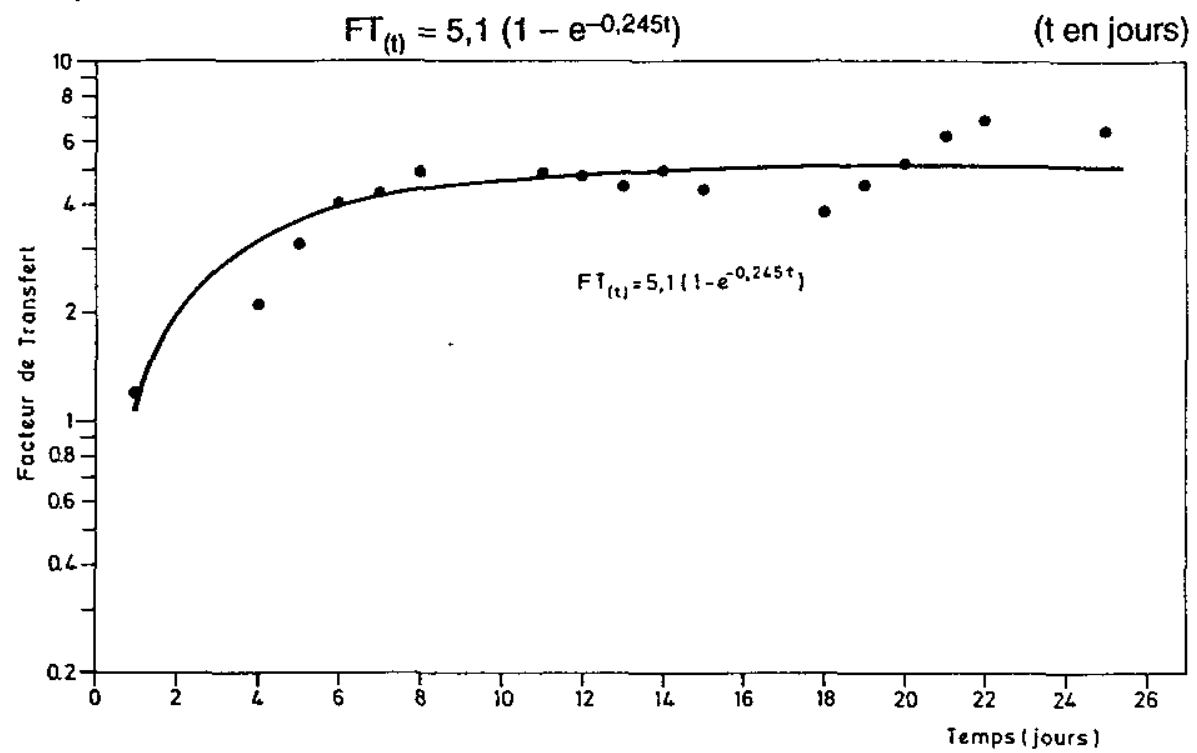

Figure 4 Evolution du facteur de transfert (FT) D. magna ( $\mathrm{Bq} g-1$ poids sec) 1 $S$. captricornutum ( $\mathrm{Bq} \mathrm{g} \mathrm{g}^{-1}$ poids sec), relativement au ${ }^{134} \mathrm{Cs}$.

Evolution of the transfer factor (FT) D. magna $\left(B q g^{-1}\right.$ dry weight) /S. ca pricornutum ( $\mathrm{Bq} \mathrm{g}^{0} \mathrm{dry}$ weight), for ${ }^{134} \mathrm{Cs}$.

Considérant une situation hypothétique où l'eau présenterait une radioactivité de $20 \mathrm{~Bq} \mathrm{ml}-1$, la conjugaison du facteur de transfert 5,1 , avec le facteur de concentration 57,4 référé en 3.1 .1 , et le facteur de concentration pour les algues $F C_{A}=1600$ (CORISCO et CARREIRO, 1990 a) permettrait de définir la situation suivante :

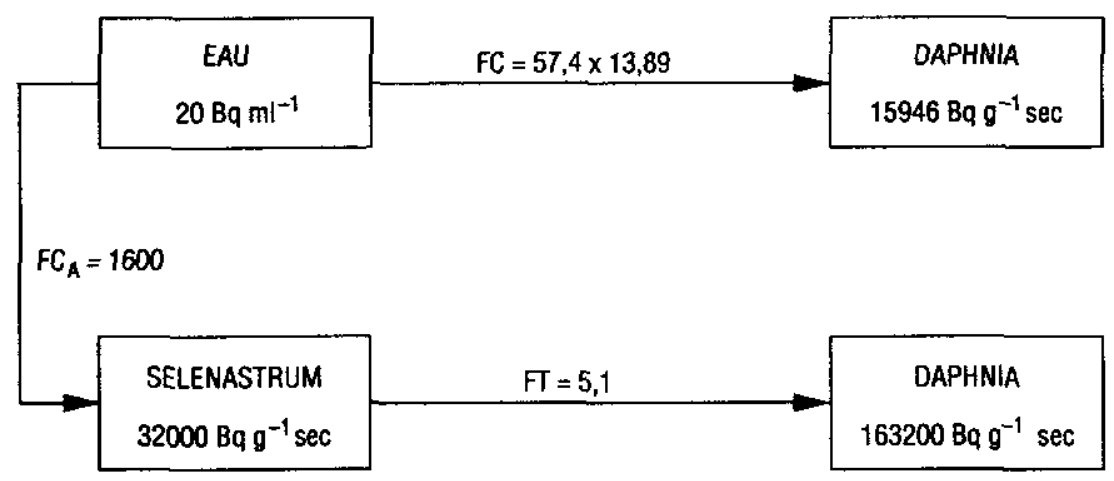

D'après ces valeurs et considérant l'hypothèse d'un effet cumulatif des deux voies de contamination, eau et nourriture, la radioactivité dans le crustacé serait approximativement $179000 \mathrm{~Bq} \mathrm{~g}^{-1} \mathrm{sec}$, étant donc de $91 \%$ la contribution de la nourriture. 
On trouve dans la littérature des expériences réalisées avec une méthodologie identique, i.e., suivant le principe de distribuer des microalgues radioactives à des crustacés planctoniques, dans un milieu non radioactif. AMIARD-TRIQUET (1979), utilisant le ${ }^{60} \mathrm{Co}$, Chlorella et Daphnia magna, réfère la valeur moyenne de 0,0019 pour le facteur de transfert trophique, calculé après avoir soumis les crustacés à un jeûne de 48 heures, suivant une période de 5 jours d'ingestion de microalgues radioactives, ce cycle se répétant pendant six semaines. GARNIER-LAPLACE (1991), utilisant $110 \mathrm{~m} A g$, Scenedesmus obliguus et $D$. magna, a conclu que la valeur maximale du facteur de transfert atteinte en un temps compatible avec la durée de vie du crustacé (30 jours) est de 0,0702 .

\subsubsection{Rétention}

La rétention du ${ }^{134} \mathrm{Cs}$ suivant une contamination par la nourriture, montre un processus différent de celui constaté lorsque la contamination est faite à partir de l'eau. En effet, une seule période biologique a été évaluée, $\mathrm{Tb}=$ 1,7 jour, l'expérience ayant duré 14 jours (fig. 5). La cinétique de l'élimination du radiocésium, considérant la variation du pourcentage de rétention, s'exprime par la fonction :

$$
R_{(t)}=100 e^{-0,410 t}
$$

(t en jours)

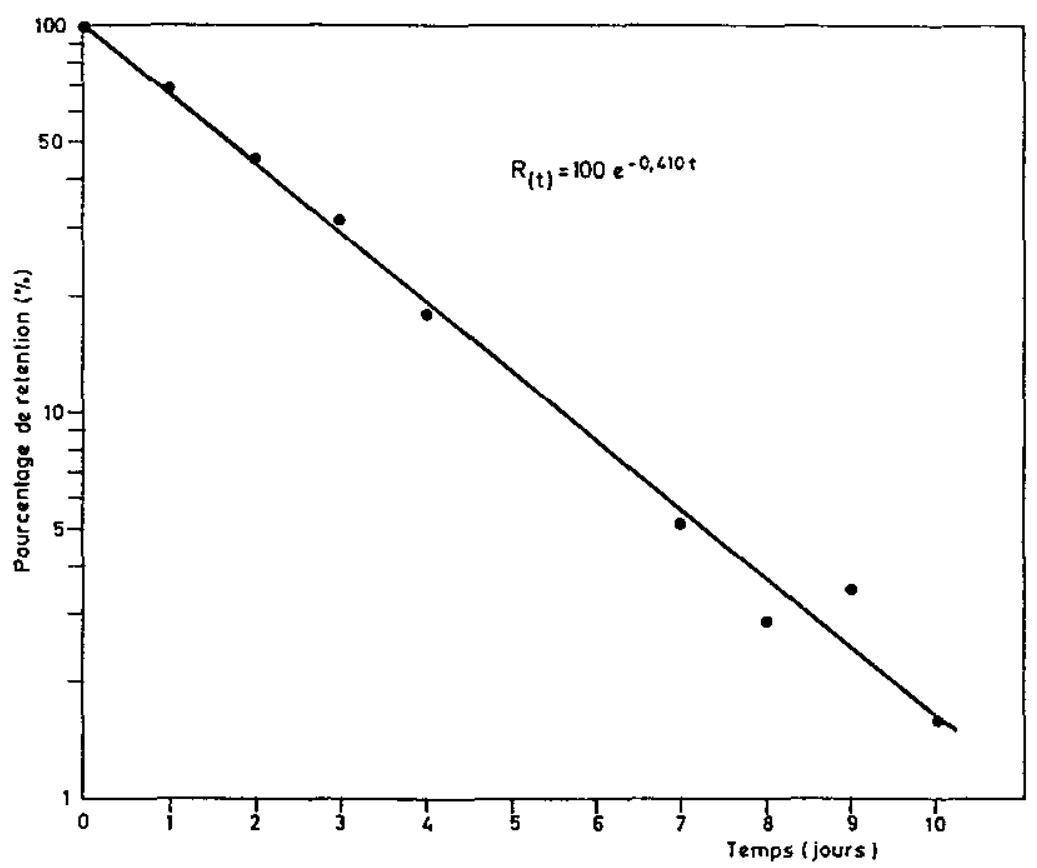

Figure 5 Evolution du pourcentage de rétention du ${ }^{134} \mathrm{Cs}$ par $D$. magna contaminée par la nourriture, montrant la seule composante exponentielle de la fonction.

Evolution of ${ }^{134} \mathrm{Cs}$ retention in D. magna contaminated from food, showing a single rate function. 
La détermination d'une seule période doit être interprétée comme l'existence d'un seul compartiment contaminé, correspondant au ${ }^{134} \mathrm{Cs}$ assimilé. Apparemment il n'y a aucun effet dû au remplacement du contenu radioactif du tube digestif en début d'expérience, par un contenu composé de microalgue non radioactives. Par ailleurs, selon Margalef (1983), le renouvellement s'effectue en 15 à 60 minutes dans les périodes actives, processus trop rapide pour pouvoir influencer cette cinétique de décontamination.

Après 24 et $72 \mathrm{~h}$ de décontamination, la rétention du ${ }^{134} \mathrm{Cs}$ chez D. magna était respectivement de $66 \%$ et $29 \%$, c'est-à-dire du même ordre que dans l'expérience qui a suivi la contamination à partir de l'eau. L'affirmation que la phase d'élimination plus lente doit correspondre à la perte du césium assimilé (voir 3.1.2), semble donc, se confirmer.

\subsection{Contamination par l'eau et la nourriture}

La figure $6 \mathrm{~A}$ montre l'évolution du facteur de concentration ( $D$. magna / eau enrichie) dans les cas où la microalgue $S$. capricornutum était présente dans le milieu contaminé et le cas de référence où la microalgue était absente. Les résultats dans le cas de référence, sont conformes à un modèle d'accumulation exprimé par la fonction :

$$
\text { FC ref } \text { (t) }_{(18,4}=1-e^{-0,46 t)}
$$

(t en jours)

La valeur à l'équilibre $(18,4)$ est plus faible que celle obtenue lors de la contamination à partir de l'eau non enrichie (3.1.1). Cette différence indique que les éléments nutritifs ajoutés à l'eau (surtout le $\mathrm{K}^{+}$), ont provoqué une compétition chimique avec le ${ }^{134} \mathrm{Cs}$.

Dans la figure $6 \mathrm{~B}$ on observe les variations du nombre de cellules algales (cell. $\mathrm{ml}^{-1}$ ) et de la concentration du ${ }^{134} \mathrm{Cs}\left(\mathrm{Bq} \mathrm{g}^{-1}\right.$ poids $\left.\mathrm{sec}\right)$ dans la microalgue. Ces paramètres se maintiennent relativement stables pendant 16 jours (cas I) et 18 jours (cas II). La moyenne arithmétique des valeurs du facteur de concentration algues / eau enrichie, vérifiés dans cette période est $\mathrm{FC}_{\mathrm{A}}=36,6 \pm 6,6$, par rapport au poids sec.

Les valeurs du FC dans les cas l et II sont plus élevées que celles dans le cas de référence, tant qu'il y a des microaigues contaminées dans le milieu. Dans le cas II, l'affaiblissement du nombre de cellules et de la radioactivité $\left(3,3 \times 10^{3}\right.$ cellules $\mathrm{ml}^{-1}$ et $46,5 \mathrm{~Bq} \mathrm{~g} \mathrm{~g}^{-1}$ au $21^{\mathrm{e}}$ jour $; 6,7 \times 10^{3}$ cellules $\mathrm{ml}^{-1}$ et $22,9 \mathrm{~Bq} \mathrm{~g} \mathrm{~g}^{-1}$ au $27^{e}$ jour) correspond à des valeurs de $\mathrm{FC}$ dans les daphnies, proches de celles prévues par le modèle de référence.

Cet essai montre que la voie trophique n'est pas aussi importante que la contamination directe par l'eau (tableau 2). Effectivement, pendant la période durant laquelle les microalgues étaient abondantes (concentration cellulaire au-dessus de $10^{6}$ cellules $\mathrm{m}^{-1}$ ) et leur radioactivité relativement stabilisée, la contribution de la nourriture à la concentration du ${ }^{134} \mathrm{Cs}$ dans $D$. magna était de $28,5 \pm 8,3 \%$, ce qui diffère beaucoup du pourcentage de $91 \%$ prévu en 3.2.1, mais il faut remarquer que les conditions expérimentales étaient différentes. 


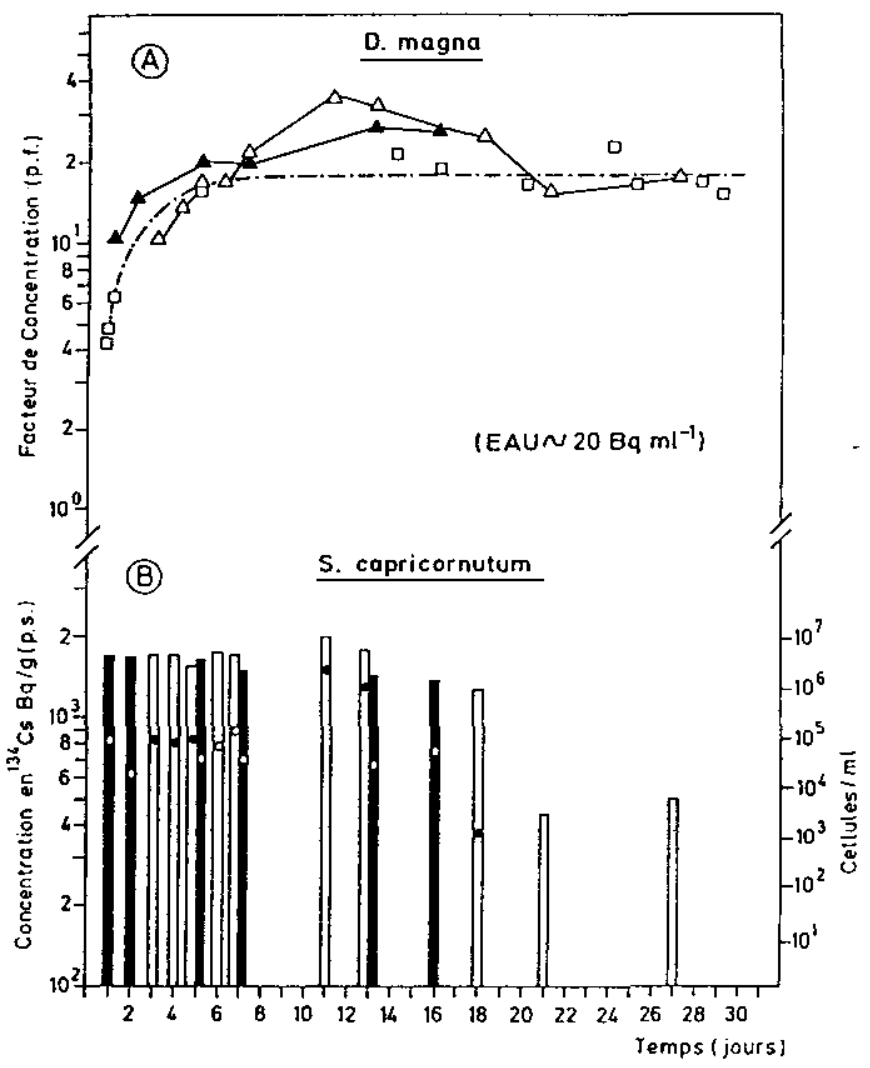

Figure 6 Contamination de $D$. magna avec le ${ }^{134} \mathrm{Cs}$, par l'eau (cas de référence) et par deux voie combinées, eau et nourriture (cas l et cas II) :

A) Evolution du facteur de concentration (FC) D. magna / eau ( $\triangle$ cas I; $\triangle$ cas II ; 口 cas de rétérence) ;

B) Variation du nombre de cellules et de la concentration du ${ }^{134} \mathrm{Cs}$ dans $S$. capricomutum (I cellules $\mathrm{ml}^{-1}$ dans le cas $\mathrm{I} ; \mathrm{T}$ cell. $\mathrm{ml}^{-1}$ dans le cas II; o radioactive des algues dans le cas 1; $\bullet$ radioactivité des algues dans le cas (I).

${ }^{134} \mathrm{Cs}$ contamination of $\mathrm{D}$. magna through the combined pathways water and food (case I and case II) and only through water (reference case): A) Evolution of the concentration factor (CF) D. magna / water (4 case 1; $\triangle$ case 11 ; $\square$ reference):

B) Variation of cells number and ${ }^{134} \mathrm{Cs}$ concentration in $\mathrm{S}$. capricornu tum ( 1 cells $\mathrm{mt}^{-1}$ in case $1 ;$ [ cells $\mathrm{mr}^{-1}$ in case $11 ; \circ$ algal radioactivity in case 11 ; $\bullet$ algal radioactivity in case (I). 
Tableau 2 Contribution (E) de la nourriture (microalgue Selenastrum capricornutum) à la contamination des daphnies (Daphnia magna) avec le ${ }^{134} \mathrm{Cs}$, par les voies combinées de l'eau et de la nourriture.

Table 2 Contribution (E) of food (microalgae Selenastrum capricornutum) to the contamination fo daphnids (Daphnia magna) with ${ }^{134} \mathrm{Cs}$ in a combined water and food pathway.

\begin{tabular}{|c|c|c|c|c|c|c|}
\hline \multirow[t]{2}{*}{ Cas } & \multirow{2}{*}{$\begin{array}{l}\text { Temps } \\
\text { (jours) }\end{array}$} & \multirow{2}{*}{$\begin{array}{c}\text { Eau } \\
\left(B q \mathrm{ml}^{-1}\right) \\
\text { A }\end{array}$} & \multicolumn{3}{|c|}{$\begin{array}{c}\text { Daphnies } \\
\text { (Bq g-1, frais) }\end{array}$} & \multirow{2}{*}{$\begin{array}{l}\text { Contribution } \\
\text { de la nourri- } \\
\text { ture (\%) (E) }\end{array}$} \\
\hline & & & $\begin{array}{c}\text { voie } \\
\text { eau (B) }\end{array}$ & $\begin{array}{l}\text { mesuré } \\
\text { (C) }\end{array}$ & $\begin{array}{c}\text { voie } \\
\text { trophique (D) }\end{array}$ & \\
\hline $\mathbf{I}$ & $\begin{array}{r}1 \\
2 \\
5 \\
7 \\
13 \\
16 \\
\end{array}$ & $\begin{array}{l}22,3 \\
23,2 \\
22,9 \\
22,8 \\
22,9 \\
23,8 \\
\end{array}$ & $\begin{array}{r}151,6 \\
257,5 \\
380,1 \\
403,6 \\
421,4 \\
437,9 \\
\end{array}$ & $\begin{array}{l}231,7 \\
343,1 \\
454,6 \\
455,0 \\
621,8 \\
611,6 \\
\end{array}$ & $\begin{array}{r}80,1 \\
85,6 \\
74,5 \\
51,4 \\
200,4 \\
173,7 \\
\end{array}$ & $\begin{array}{l}34,6 \\
24,9 \\
16,4 \\
11,3 \\
32,2 \\
28,4 \\
\end{array}$ \\
\hline II & $\begin{array}{r}3 \\
4 \\
5 \\
6 \\
7 \\
11 \\
13 \\
18\end{array}$ & $\begin{array}{l}24,4 \\
23,2 \\
22,2 \\
23,1 \\
22,5 \\
21,5 \\
22,5 \\
23,7\end{array}$ & $\begin{array}{l}336,7 \\
359,6 \\
368,5 \\
397,3 \\
398,3 \\
393,5 \\
414,0 \\
436,1\end{array}$ & $\begin{array}{l}258,2 \\
321,8 \\
366,4 \\
387,9 \\
498,6 \\
769,2 \\
722,2 \\
586,7\end{array}$ & $\begin{array}{c}C<B \\
C<B \\
C<B \\
C<B \\
100,3 \\
375,8 \\
308,2 \\
150,6\end{array}$ & $\begin{array}{c}- \\
- \\
- \\
- \\
20,1 \\
48,9 \\
42,7 \\
25,7\end{array}$ \\
\hline
\end{tabular}

A : concentration du $134 \mathrm{C}$ s dans l'eau.

B : calcul de la radioactivité pressente dans les daphnies, due à la contamination par l'eau, en utilisant le modêle prédicti du facteur de concentration (voir 3.3). B = A X 18.4 (1-e-0,46t).

C: concentration du ${ }^{134} \mathrm{Cs}$ mesuré dans les daphnies.

$D$ : calcul de la radioactivité des daphnies, due au transfert trophique. $D=C-B$.

$E: D / C \times 100$.

En utilisant les résultats obtenus dans notre expérience de contamination globale, on peut établir la situation suivante :

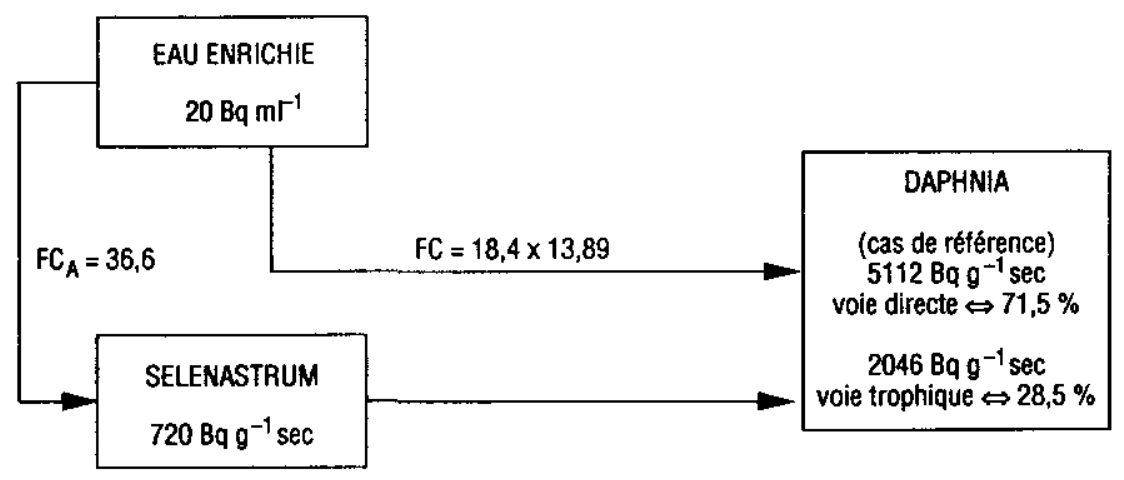


Le facteur de transfert trophique calculé à partir de ces résultats est $\mathrm{FT}^{\prime}=$ $2046 \div 720=2,8$. Bien que un peu plus faible que celui calculé au paragraphe 3.2.1, il est du même ordre de grandeur.

LAMBRECHTS et al. (1984) utilisant un protocole expérimental pareil, ont calculé en $80 \%$ la contribution de la nourriture (Cyanophycées et Diatomophycées) dans la contamination des Copépodes par l'eau et la nourriture, avec les radionucléides ${ }^{137} \mathrm{Cs}$ et $65 \mathrm{Zn}$. GARNIER-LAPLACE (1991), a calculé en $42 \%$ la contribution de la microalgue Scenedesmus obliquus dans la contamination globale de Daphnia magna avec le ${ }^{110 \mathrm{~m}} \mathrm{Ag}$.

\section{4 - CONCLUSIONS}

La contamination simultanée des daphnies par l'eau et la voie trophique, semble mener à la conclusion que la voie directe serait la plus importante, puisque la nourriture ne contribuerait que avec $29 \%$ dans la contamination globale.

Cependant, le calcul effectué à partir des fonctions issues des données des expériences de contamination par la voie directe et la voie trophique séparément, indique que cette voie serait prépondérante.

En conclusion, la prépondérance d'une voie sur l'autre dépendrait vraisemblablement des conditions expérimentales, tel que l'on a déjà discuté. L'importance relative des voie de contamination doit donc être soigneusement évaluée, en essayant de faire une approximation aussi meilleure que possible, des conditions expérimentales aux conditions vérifiées dans l'environnement.

Etant donné l'importance des daphnies comme maillon dans les chaînes trophiques d'eau douce, le facteur de concentration (57 \pm 3 , à l'équilibre) ,et surtout le facteur de transfert trophique $(5,0 \pm 0,6$, à l'équilibre), pourraient conduire à une contamination assez significative, en ce qui concerne le ${ }^{134} \mathrm{Cs}$.

La comparaison des deux courbes de rétention met en évidence que le compartiment correspondant à la période biologique plus longue, $\mathrm{Tb}_{2}=$ 1,6 jour, dans l'expérience où la contamination a été réalisée par l'eau, est tout à fait semblable au compartiment unique, $\mathrm{Tb}=1,7$ jour, évalué après l'ingestion de nourriture contaminée. Cette composante de rétention peut être attribuée, dans les deux expériences de désorption, à la perte du césium assimilé.

D'après ces résultats, l'assimilation semble être le mécanisme de concentration du ${ }^{134} \mathrm{Cs}$, le plus important dans le crustacé Daphnia magna. 


\section{RÉFÉRENCES BIBLIOGRAPHIQUES}

AMIARD TRIQUET C., 1979. Modalités de la contamination de deux chaînes trophiques dulcaquicoles par le cobalt-60. III: Contamination des organismes par l'intermédiaire de la nourriture. Water, Air and Soil Pollution, 12, 155-170.

CARREIRO M.C.V., 1990. Ecological study of three sampling stations in Tejo River. LNETI/DPSA - $n^{\circ} 4$ (III série).

CORISCO J.A.G., CARREIRO M.C.V., 1990, a. Etude expérimentale sur l'accumulation et la rétention du ${ }^{134} \mathrm{Cs}$ par une microalgue planctonique, Selenastrum capricornutum Printz. Revue des Sciences de l'Eau, 3, 4, 457-468.

CORISCO J.A.G., CARREIRO M.C.V., 1990, b. ${ }^{134} \mathrm{Cs}$ Transfer from water and food to the Cyprinid Tinca Tinca Linaeus : Uptake and Loss Kinetics. Presented on the Seminar on Comparative Assessment of the Environmental Impact of Radionuclides Released during three major Accidents : Kyshtym, Windscale, Chernobyl. Luxembourg, 1-5 october 1990.

DE PAUW N., LAUREYS P., MORALES J., 1981. Mass Cultivation of Daphnia magna Straus on Ricebran. Aquaculture, 25, 141152.

FOULQUIER L., GRAUBY A., LAMBRECHTS A. 1978. Absorption directe et transfert du césium-137 dans une chaine alimentaire d'eau douce simplifiée. Verh. Internat. Verein. Limnol., 20, 1992-1998.

GARNIER-LAPLACE J., 1991. Transfert au consommateur primaire pélagique : la Daphnie. In: Etude des mécanismes de transfert de largent - $110 \mathrm{~m}$ en eau douce. Rapport CEA-R-5549, p. 85-92.

KING S.F., 1964. Uptake and Transfer of Cesium-137 by Chlamydomonas, Daphnia and Bluegill Fingerlings. Ecology, 45, 4, 852-859.

LAMBRECHTS A., FOULQUIER L., BAUDIN J.P., 1984. Données expérimentales sur le rôle du sédiment et de microorganismes dans le transfert de radionucléides en eau douce. In : Role of microorganisms on the behaviour of radionuclides in aquatic and terrestrial systems and their transfer to man. Proceedings of a workshop held in Brussels, 25-27 April 1984, in cooperation with the Institut Royal des Sciences Naturelles de Belgique. Editors: E. Bonnyns-Van Gelder \& R. Kirchmann, p. 139-152.

LAMBRECHTS A., 1984. Essai de modélisation du transfert du césium-137 dans les compartiments d'un écosystème d'eau douce simplifié. Papport CEA-R-5268, $181 \mathrm{p}$.

MARGALEF R., 1983. Limnologia. Ediciones Omega, S.A., Platon, 26, Barcelona-6, $1010 p$.

REIS M.F., FREITAS, M.C. CARREIRO M.C.V., MARTINHO E., 1988. Analise Multielementar de Peixes e Agua do Rio Tejo pela Tecnica da Analise por Activação com Neutroōes. $1^{\text {a }}$ Conferencia Nacional sobre a Qualidade do Ambiente. Aveiro (Portugal) 22-24 Fevereiro 1988. LNETI/DPSR-B$n^{\circ} 104$.

WHICKER F.W., SCHULTZ V., 1982. Radioecology : nuclear energy and environment. CRC Press, Inc., Boca Raton, Florida, vol. II, $228 \mathrm{p}$. 